\title{
Conceptual Framework for the Analysis and Modeling of GNSS Measurements based on Gaussian Process
}

\author{
R.S. Nahar, K.M. Ng, N.A. Razak, and J.Johari
}

\begin{abstract}
The Global Navigation Satellite Systems (GNSS) have been used in autonomous vehicles and remote sensing. The GNSS receiver can be a conventional device or an enhanced device that adopts precise positioning technology such as differential positioning and real time kinematics. However, the GNSS devices could still produced outputs that are subjected to various sources of errors. Hence, their performance needs to be evaluated and analyzed. Subsequently, error mitigation techniques are proposed to enhance the GNSS performance. In this paper, we aim to present a conceptual framework on the analysis and modeling of GNSS measurements based on Gaussian Process (GP). Firstly, the methods of performance analysis of GNSS devices are presented. Secondly, current works on the applications of GP to model GNSS and position sensors' errors are briefly reviewed. Subsequently, we present a conceptual framework to provide an overview to the readers the purpose of various performance evaluation methods. On the other hand, the conceptual framework on the current applications of GP to model and improve GNSS errors is presented to wrap up the concepts and methods of GP that has been implemented by researchers thus far. The established framework assists us to identify some research gaps and further works that can be explored in the applications of Gaussian process to model GNSS measurements and errors.
\end{abstract}

Index Terms - Deep Gaussian process, Gaussian Process, Global navigation satellite systems

\section{INTRODUCTION}

A UTONOMOUS vehicles navigation relies greatly on various sensors such as light detection and ranging (LIDAR), vision system, communication devices, vehicle odometry, global positioning system (GPS) and inertial measurement sensors in ascertaining the vehicle location accurately. To date, various localization technologies have been developed to ascertain the vehicle pose and location [1-3]. The ultimate purpose of these proposed methods is to achieve localization of vehicle current pose and location with high degree of accuracy.

This manuscript is submitted o28 April 2021 and accepted on 5 July 2021. R.S. Nahar, K.M. Ng, N.A. Razak and J.Johari are with the School of Electrical Engineering, College of Engineering, Universiti Teknologi MARA, 40450 Shah Alam, Selangor (e-mail: ngkokmun@uitm.edu.my)

1985-5389/C 2021 The Authors. Published by UiTM Press. This is an open access article under the CC BY-NC-ND license (http://creativecommons.org /licenses/by-nc-nd/4.0/).
The GPS is one of the sensor employed to ascertain a vehicle or user position on earth. In a wider sense, the GPS is part of the Global Navigation Satellite Systems (GNSS) that includes Europe's Galileo, the USA's NAVSTAR, Global Positioning System (GPS), Russia's Global'naya Navigatsionaya Sputnikovaya Sistema (GLONASS) and China's Beidou satellite systems [4]. A conventional GNSS receiver (i.e. Receiver 1 and 2 respectively in Fig. 1) is used to determine the user position anywhere on earth. The position of a receiver is determined using a triangulation method based on measurements of pseudo-distances and coded signals from at least three known satellite positions. For more accurate positioning, the conventional receivers can receive signal combination from more than three satellites and mitigate errors using different strategies.

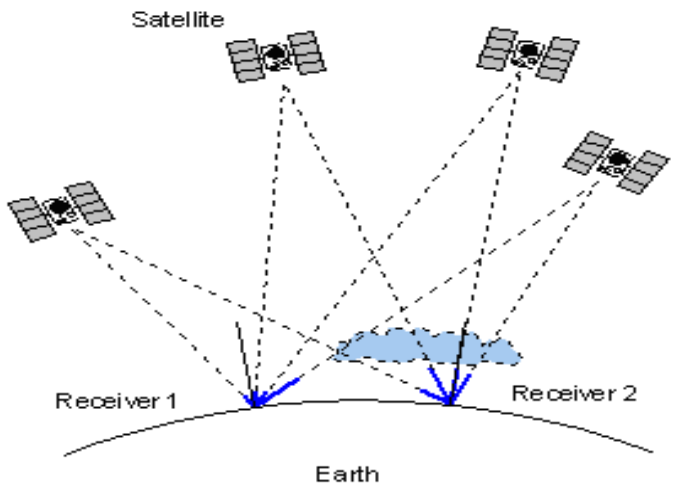

Fig. 1. GNSS positioning system [4]

The conventional GNSS positioning errors are normally caused by atmospheric, satellite clock errors and noise. An L1single frequency receiver could produced horizontal errors ranging from 5 to 20 meters [5]. To improve accuracy in conventional GNSS, differential GNSS (dGNSS) shown in Fig. 2 can be utilized. It comprised of a receiver (base station) on a precisely known location. The base station compares the ranges from satellites in view and calculates range errors. The error corrections are sent to other receivers (rovers) through radio or other links. The corrected ranges are used by the rover to determine its position. The real time kinematics (RTK) GNSS also operates based on the same principle. The dGNSS uses 
pseudo-range measurements whereas RTK uses carrier phase shift measurements.

The RTK-GNSS can be categorized to both medium-high cost [7] and the low-cost RTK-GNSS [8] that could achieve centimetres positioning. However, performance evaluation on five different brands of low-cost $(<\$ 1000)$ RTK-GNSS (i.e. Swift Piksi Multi, NVS Technologies NV08C-RTK, Emlid Reach, u-blox NEO-M8P and Skytraq S2525F8-RTK) operating in rural, sub-urban and urban landscapes has been conducted in [8]. Each of the low-cost RTK-GNSS was tested under these landscapes using a Navcom and a Patch antenna respectively. The results reveal that under dynamic testing (i.e. when the rover is moving) in these landscapes; they cannot hold an RTK fixed-integer solution for any significant time in dynamic applications. For example, the accuracy results for sub-urban condition ranges between $0.4 \mathrm{~m}$ (Reach) to $3.5 \mathrm{~m}$ (NEO-M8P) when using the Navcom antenna. When using the Patch antenna, the highest accuracy is achieved by Reach at $0.05 \mathrm{~m}$ whereas the Skytraq recorded accuracy at $5 \mathrm{~m}$ with the same antenna.

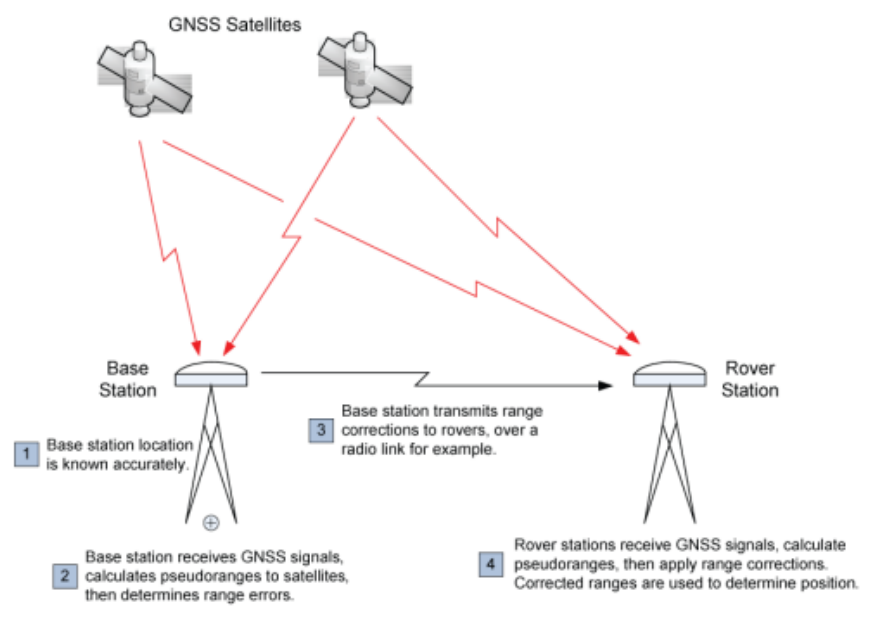

Fig. 2. Differential/RTK GNSS positioning system [5]

Many of the sensors employed in the localization systems may have errors i.e. single point data from GNSS and drifts in inertial measurement systems. In assisting these localization systems; research on improving sensor measurements from GNSS and inertial navigation system (INS) has been an area of research focus. The improved sensor measurements will in turn improve the overall performance of a localization system when the measurements are fused with data from other sensors [9-11].

In view of the inaccuracies in low-cost RTK-GNSS [8] and conventional GNSS [5]; there is a need to improve and optimize measurements from these devices. Hence, this paper aims to study analysis methods in GNSS measurements. Secondly, the authors aim to study related literatures specifically on modeling errors from position sensor measurements using Gaussian process (GP). At the end of the brief review, a conceptual framework for analyzing and modeling errors in GNSS based on previous works is presented and discussed. Finally, research gaps and future works for the analysis and modeling of GNSS errors based on GP is proposed in this paper. Section 2 of this paper outlines related works in the analysis of GNSS measurements. Section 3 reviewed previous works on modeling errors using GP. Section 4 outlines the findings and discuss the conceptual framework for the analysis and modeling of GNSS errors based on GP. This section also highlights the research gaps and provide directions for future research.

\section{PERFORMANCE ANALYSIS OF GNSS RECEIVER MEASUREMENTS}

The position of an object on earth surface could be obtained by a GNSS receiver that can collects data from all the satellite systems. However, the accuracy of positioning depends largely on factors such as landscape, weather conditions, satellite position, and receiver quality. In recent years, various technologies such as Precise Point Positioning (PPP), Satellitebased Augmentation System (SBAS) and real time kinematics (RTK) have been implemented to improve the accuracy of positioning in GNSS receivers.

\section{A. Performance Evaluation of GNSS Measurements}

The performance of these conventional and RTK-GNSS receivers could be evaluated in terms of accuracy, integrity, continuity and availability [12]. Accuracy is defined as the difference between the measured and actual position, speed or time. Integrity is the ability of the receiver to provide a threshold of confidence in its measurements. A receiver with good continuity is evaluated based on its ability to function without interruption. Finally, availability is the amount of time the measured signals are obtained with high accuracy, integrity and continuity. These are normally done with reference to some ground truth data.

Demoz Gebre-Egziabher [8] evaluated five different types of low-cost RTK-GNSS namely Piksi Multi, NVS Technologies NV08C-RTK, Emlid Reach, u-blox NEO-M8P and Skytraq S2525F8-RTK. The performances of these RTKGNSS were evaluated in terms of accuracy, continuity and availability using two types of antenna. The accuracy is defined as the accuracy of horizontal positioning in meters whereas continuity was assessed based on the number of times an RTK fixed-integer solution was lost per RTK minute. Availability is the percent of time a receiver recorded an RTK fixed solution. These devices were subjected to both static and dynamic tests under rural, sub-urban and urban environments. Rural areas have a clear view of the sky with no obstructions or nearby metal structures. Urban areas have tall, metal structures and a narrower view of the sky from the antenna's point-ofview. Sub-urban areas fall somewhere in-between. The performance of low-cost RTK-GNSS was compared to SF3050 (high-end) and Eclipse P307 (mid-range) that have superior performance in terms of accuracy, continuity and availability. It was observed that the Emlid Reach has the best performance among the low-cost RTK-GNSS.

Luo et al. [13] evaluated the performance of Leica Viva RTK-GNSS receivers based on availability, accuracy, coordinate quality (CQ) indicator and time to fix (TTF). The Leica Viva GS10 with AS10 antenna was used as base station whereas Leica GS15 was used as rover. Static test was conducted on the rover signals (operating at short baseline) placed under the canopy and open-sky situations. The received 
signals were evaluated on the accuracy, availability, CQ indicator and TTF when the receiver operates using a GPS only, GLONASS only, Beidou only and combination of GPS, GLONASS and Beidou (GGB). In general, the open-sky tests reported better performances in availability, accuracy, CQ indicator and TTF compared to canopy test. The receiver recorded the lowest performance in availability, accuracy, CQ indicator and TTF when operating using only Beidou signals compared to GPS only, GLONASS only and GGB. Overall, the best performances were observed in GGB signals where RTK fix accuracy is 0.015 and $0.005 \mathrm{~m}$ for canopy and open sky respectively. The RTK fix availability for GGB was $99.99 \%$ with the lowest mean TTF.

Baybura et al. [14] evaluated the performance of long base RTK (LBRTK) and network RTK (NRTK) using the accuracy of positioning. The LBRTK comprised of a a base station that employed Ashtech Proflex 800 GNSS receiver and AERAT1675_120 SPKE geodetic antenna. Long base RTK could project corrections to six static stations that were located between $5 \mathrm{~km}$ to $60 \mathrm{~km}$ from the base station. These static stations employed the Stonex S9 GNSS. On the other hand, these six stations can also receive corrections from NRTK via the CORS-TR network. The authors compared the measurements' accuracy which is determined in terms of standard deviation from the mean value for NRTK and LBRTK respectively. Deviations increased when the static station move away from the base in LBRTK. On the other hand, the NRTK has better performance in accuracy compared to the LBRTK. In addition, the work also evaluated the effects of dilution of precision and ionospheric errors.

\section{B. Performance Evaluation of GNSS Signal Qualities}

Another type of performance evaluation is conducted to evaluate GNSS signal qualities due to multipath and signal obstruction. This is due to GNSS broadcasted signals are subjected to multipath errors due to reflection and diffraction by metallic objects, lakes and other reflecting surfaces. Hence, the multipath causes reception of direct GNSS signal together with the reflected signals simultaneously. Multipath will result in errors in pseudo-range measurement and carrier phase. Another source of error in GNSS is the weak geometric configuration of satellites where the distribution of satellite in the view of the observer is obstructed by house/building wall and tree canopies. This will cause an increased in the dilution of precision (DOP).

Chengyan et al. [15] studied the multipath performance and its impact on the positioning performance of received GNSS signal from Beidou satellite system (BDS). The signals received from BDS at a static GNSS receiver (with a $25 \mathrm{~m}$ high attenna) at an observatory station was analysed using mathematical verifying model to determine the pseudo-range multipath error; waveform and modulation error and the correlation characteristics. This study served as reference for BDS future signal design and system construction. Rychlicki et al. [16] developed a software that can log and process GNSS signals from multiple GNSS receivers simultaneously. The software was used to process GNSS signals and evaluate positioning accuracy in terms of average numbers of visible satellites, dilution of precision (DOP), horizontal dilution of precision
(HDOP) and vertical dilution of precision (VDOP). The GNSS signals was collected in 4 types of test scenarios i.e. i) Mode 1: Static (conventional) in an open area; ii) Mode 2: dynamic (real time kinematic - RTK) at minimum speed of $100 \mathrm{~km} / \mathrm{h}$ on a dual carriage way; iii) Mode 3: dynamic (RTK at maximum speed of $50 \mathrm{~km} / \mathrm{h}$ in a built up area and Mode 4: static in a built up area. All nine GNSS receivers evaluated in this work demonstrated different performances where some GNSS receivers were more superior than others due to the dual frequencies technology. In general, the average number of satellites were higher in mode 2, 3 and 4, which were located in built up area (some obstruction from building and structures). This is also due to partial obstruction of the satellite signals by the vehicle. All GNSS receivers demonstrated highest DOP, HDOP and VDOP in Mode 1 compare to other modes. Overall, all receivers recorded values lower than 2 threshold, for DOP, HDOP and VDOP which indicates correct positioning.

Luo et al. [13] also evaluated RTK-GNSS signals based on mean geometric dilution of precision (GDOP) for the Leica GNSS operating using signals from GPS only, GLONASS only, Beidou only and combination of GPS, GLONASS and Beidou (GGB). It was found that the Beidou only setup reported the worst GDOP. On the other hand, the GGB setup demonstrated the best GDOP compared to GPS, GLONASS and Beidou only. The work also analysed the signal-to-noise ratio for the reception of the GPS only, Beidou only and GLONASS only signals

Guo et al. [17] evaluated raw GNSS signals from Xiaomi 8 smartphone which operated in dual frequencies namely the L1/E1 single-frequency and L5/E5 frequency. The newly added L5/E5 signals with binary phase-shift keying (BPSK(10)) and alternative binary offset carrier (AltBOC $(15,10)$ ) modulation were found to be less prone to distortions from multipath reflections compared to L1/E1 [18,19]. Guo et al. [17] analysed characteristic of raw multi-GNSS observations from Xiaomi Mi 8 under static open and dynamic complex environments (mixture of open area and built up area). The Xiaomi 8 received RTK corrections from a base station that employed Septentrio PolaRx 5 receiver connected with a Trimble antenna. The raw GNSS measurements from the L1/E1 and L5/E5 frequencies respectively were evaluated in terms of carrier-to-noise ratio, noise of pseudo-range, carrier phase observations, the percentage of pseudo-range gross errors and carrier phase cycle slips. It was observed that when the carrier-to-noise ratio increases, the standard deviation (SD) of the pseudo-range residuals decreases. The L5/E5 measurements demonstrated lower SD pseudorange residuals below $5 \mathrm{~m}$ whereas L1/E1 measurements could reach up to $15 \mathrm{~m}$. The carrier phase noise was below $2 \mathrm{~cm}$ and the phase cycle slips increases in dynamic GNSS-degraded environments.

GNSS signals had been studied and evaluated using mathematical simulations. He et al. [20] introduced statistical channel model that considers statistical distributions of various geometric path delays and multipath relative amplitudes. The model could compute multipath error envelopes which are valid for static test of GNSS under various multipath environments such as open, rural, suburban and urban scenarios where various signals in the modulation scheme such as BPSK, MBOC and BOC were analysed using the model. 
TABLE I

GNSS PERformance EVAluation Methods

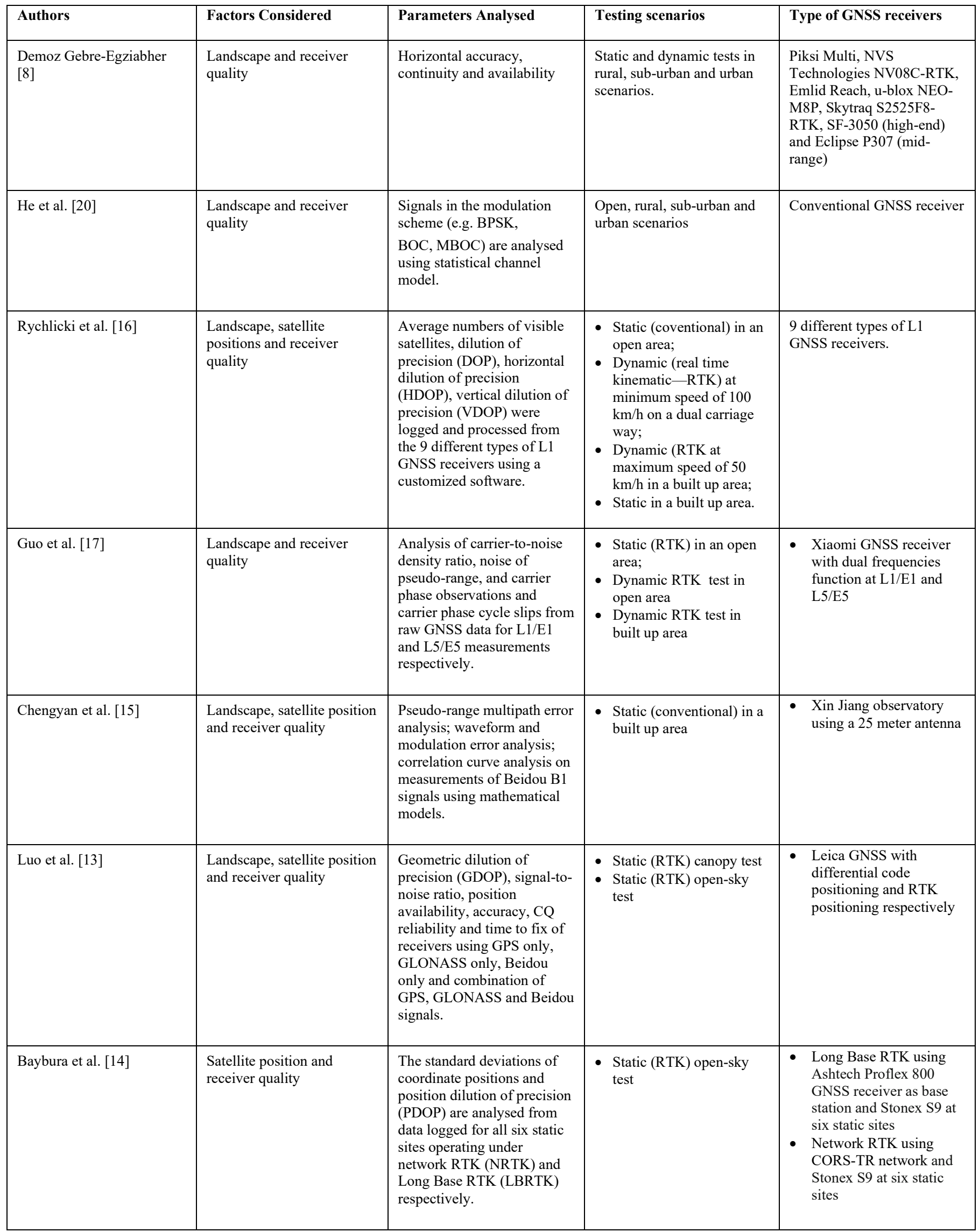


The performance evaluation methods of GNSS (conventional and RTK) were summarized in Table 1. This review is not exhaustive but is sufficient to illustrate performance analysis of GNSS receivers at the signals and device outputs respectively. Table 1 illustrated that, when performing performance evaluation; the factors and parameters affecting the GNSS measurements were defined, selected and evaluated. These measurements were normally collected under various testing scenarios; such as static and/or dynamic tests in rural, urban or sub-urban environments. The performance is also dependent on the receiver and antenna quality used, that can be categorized to high-cost, mid-range and low-cost receivers.

\section{APPLICATIONS OF GAUSSIAN PROCESS IN MODELING ERRORS IN GNSS SENSORS}

Gaussian process (GP) model correspond to a random function with joint multivariate Gaussian distribution. Cheng et al. [21] found that the GP model is suitable for data that has correlated outcome values, functional (time-varying) covariates, nonlinear and non-stationary effects, and model inference. GP is based on a Bayesian non-parametric model that naturally provide a prior for an unknown regression function $f(X)$ estimated from input data $X$ and observation $y \in \mathbb{R}$ shown in (1). The $y$ differs from $f(X)$ by additive noise defined as $\varepsilon=$ $\mathcal{N}\left(0, \sigma_{\mathrm{n}}^{2}\right)$, is the zero-mean Gaussian noise with variance $\sigma_{\mathrm{n}}^{2}$.

$$
y=f(X)+\varepsilon
$$

The $f(X)$ is uniquely specified by a mean function $\mathrm{m}(t)=0$ and a covariance function $k\left(x, x^{\prime}\right)$ shown in (2). The GP specifies $f(X)$ using $X=\left(\mathrm{x}_{1}, \mathrm{x}_{2}, \ldots, \mathrm{x}_{\mathrm{N}}\right)$ (where $\mathrm{x}_{\mathrm{i}} \in \mathbb{X}$ ) finite number of fixed inputs and corresponding observations $y=\left(y_{1}, y_{2}, \ldots, y_{\mathrm{N}}\right)$.

$$
f(X) \sim \mathcal{G} \mathcal{P}\left(0, k\left(x, x^{\prime}\right)\right)
$$

GP could estimate priori distribution for the entire estimated non-linear function. The prior density function $f \mid X$ with zero mean is shown in (3) where $\boldsymbol{K}$ is an $N \times N$ covariance matrix defined by $\boldsymbol{K}=\mathrm{k}\left(\mathrm{x}_{\mathrm{i}} ; \mathrm{x}_{\mathrm{j}}\right)$. Equation (4) defined the output density function $y \mid f(X)$.

$$
\begin{gathered}
f \mid X \sim \mathcal{N}(0, K) \\
y \mid f(X)=\mathcal{N}\left(f(X), \sigma_{\mathrm{n}}^{2}\right)
\end{gathered}
$$

Recently GP model has been applied to model and improve errors in position sensors. In robot motion planning, odometry sensors were used to feed initial state measuremet in SLAM sensor. As the odometry may have errors, this may affect the accuracy of the initial state measurement fed into the SLAM. To solve this problem, Carrio et al. [22] trained a Gaussian process on odometry residuals to model poor traction performance. The methodology trains a GP on the residual between the non-linear parametric motion model and the ground truth training data. The results show that the GPR improved initial states from the odometry successfully resulting in enhanced visual Simultaneous Localization and Mapping
(SLAM). In another similar work, GP was directly applied to RGBDSLAM to represent the measurement noise of the position data from the SLAM [23]. GP models the independent, identically distributed (i.i.d) Gaussian noise based on an exponential kernel with hyper parameters estimated with the ML-II method [24]. The GP was extended to a Kalman filtering method to improve localization performance of the RGBDSLAM.

In the area of positioning using GPS, Ye et al. [25] used Noisy Input Gaussian Process Regression (NIGPR) and an enhance Kalman filter to correct position and orientation errors in airplane during GPS out stages. In another work, GP has been applied to model GPS errors from buses that were equipped with GPS sensors that collect vehicle trajectory data. The GPS sensors in buses may be subjected to errors due to environmental, distinct calibrations and time. Hence, this causes variances in the buses positions and time stamps even when they travel in the same trajectory. Kortesalmi [26] solved the GNSS variance estimation problem along a bus trajectory by means of a mixture of GP trained using GNSS positions and time stamp data. The result is a grid where each point has a longitude and latitude mean and variance. In the same work, a mixture of Gaussian processes that were trained with time stamp and speed information of buses is also used to predict the arrival time for public transportation buses. The arrival time prediction is from one bus stop to the next, not for the whole trajectory. Similar works using GPs have been used with spatiotemporal datasets to create local trajectory models [27-29]. In [27], GPs are used to recognise common activities from spatiotemporal datasets. In [28], sparse GP regression is used to perform trajectory modelling.

The majority of the noise in GPS signal is the signal delay caused by the ionosphere which can be described by the vertical Total Electron Content (vTEC) [30]. The vTEC contributes the majority of noise in the GPS signal received by ground stations. To eliminate this delay, a GP model is learned to predict the vTEC value for any given geographic coordinate [30]. Data was collected from 16 RTK-GNSS over a period of 10 days. Experiments were conducted using the first 5 days of data to train the GP model in an offline manner whereas the next 5 days data was used to test the model. An algorithm to detect outliers and split the received GPS signal was developed to separate the signals into clean and noisy subsets. The GP model is then trained on the clean dataset to minimize a loss function based on an exponential kernel function. The learned GP model was applied to the noisy data set to compute absolute residuals. Next, a Filter-Reweight-Retrain (FRR) function optimization algorithm was proposed to truncate the noise level in the noisy data set based on the absolute residuals predicted. It was able to filter large residuals from the noisy data. The remaining noisy data points are reweighted according to their residual error and finally the GP model is retrained on the reweighted noisy data points and the clean dataset together. A successfully optimized GP model was applied to a testing station to predict the vTEC and the double difference related to the testing station for successful positioning. The method exhibit a high ratio of 
successful positioning compared to other existing optimizing algorithms.

TABLE II

SUMMARY OF SOME GP METHODS

\begin{tabular}{|c|c|c|}
\hline Authors & Methods & Results \\
\hline Carrio et al.[22] & $\begin{array}{l}\text { GP trained using } \\
\text { odometry residuals } \\
\text { with various kernel } \\
\text { functions i.e. radial } \\
\text { basis function (RBF), } \\
\text { RBF+linear, matern } \\
5 / 2 \text { and matern } \\
5 / 2+\text { linear. }\end{array}$ & $\begin{array}{l}\text { The GP trained using } \\
\text { RBF has the best } \\
\text { performance in } \\
\text { predicting odometry } \\
\text { residuals with RMSE } \\
\text { of } 0.004494 \text { and MAE } \\
\text { of } 0.002127\end{array}$ \\
\hline Ye et al. [25] & $\begin{array}{l}\text { GP trained to model } \\
\text { GPS errors and feed to } \\
\text { a Kalman Filter (KF) } \\
\text { to estimate all inertial } \\
\text { navigation system } \\
\text { (INS) errors in an } \\
\text { aeroplane's position } \\
\text { and orientation } \\
\text { system. }\end{array}$ & $\begin{array}{l}\text { Produce } 90 \% \text { better } \\
\text { estimate compared to } \\
\text { only using KF. }\end{array}$ \\
\hline Kortesalmi [26] & $\begin{array}{l}\text { Gaussian processes } \\
\text { trained using GNSS } \\
\text { positions and time } \\
\text { stamp data to estimate } \\
\text { position variance and } \\
\text { bus arrival time along } \\
\text { a bus route. }\end{array}$ & $\begin{array}{l}\text { The variance variation } \\
\text { is larger at certain } \\
\text { locations on the bus } \\
\text { line i.e. sharp } \\
\text { trajectory turn due to } \\
\text { poor GP modelling. } \\
\text { The root mean square } \\
\text { error for predicted } \\
\text { arrival time ranged } \\
\text { from } 5 \text { to } 19 \text { seconds. }\end{array}$ \\
\hline Lin et al. [30] & $\begin{array}{l}\text { GP with exponential } \\
\text { kernel function and } \\
\text { FRR algorithm to } \\
\text { predict vTEC and } \\
\text { double difference } \\
\text { matrix at static GNSS } \\
\text { station }\end{array}$ & $\begin{array}{l}\text { The GP with FRR } \\
\text { reported an average } \\
\text { ratio of RTK } \\
\text { positioning at } 78.4 \% \\
\text { which has the best } \\
\text { performances } \\
\text { compared to another } \\
\text { four optimization } \\
\text { algorithms that } \\
\text { perform at } 70 \% \text { and } \\
\text { below. }\end{array}$ \\
\hline $\begin{array}{l}\text { Hines and Hetland } \\
{[31]}\end{array}$ & $\begin{array}{l}\text { Predict slow slip } \\
\text { events using GP } \\
\text { trained using } \\
\text { Wendland covariance } \\
\text { function where } \\
\text { covariance function } \\
\text { parameters were } \\
\text { constrained with } \\
\text { maximum likelihood } \\
\text { methods. }\end{array}$ & $\begin{array}{l}\text { Good fitting between } \\
\text { observed and } \\
\text { predicted data was } \\
\text { found in two out of } \\
\text { four ground stations. } \\
\text { Misfit between } \\
\text { observed and } \\
\text { predicted data in two } \\
\text { stations is likely due to } \\
\text { oversmoothing. }\end{array}$ \\
\hline
\end{tabular}

Transient strain from GNSS data can be used to study geophysical processes and seismic activities on earth surface. Hines and Hetland [31] proposed Gaussian Process Regression (GPR) to estimate transient strain from GNSS data. GPR was used to detect transient strain resulting from slow slip events (SSE) in the Pacific Northwest. The GPR was described with addition of the temporally correlated Gaussian noise described using first order Gauss-Markov process (FOGM). Thus, maximum likelihood methods are used to determine the parameters that needs to be constrained in the covariance function. The temporal covariance of the model is described by a compact Wendland covariance function. This was reported to have significantly reduces the computational burden that can be associated with the GPR.

Selected works mentioned above are summarized into Table 2. The results demonstrated GP can be used to predict errors from position and GNSS sensors. Some works [22, 25, 30] reported promising results whereas inferior performance were found in $[26,31]$ due to poor modeling and oversmoothing.

\section{FINDINGS AND DISCUSSION}

\section{A. Findings and Discussion on GNSS Performance Evaluation and Analysis}

The performance evaluation of GNSS measurements based on accuracy, availability, continuity and integrity serve as general indicators on the overall quality of measurements produced by both conventional and RTK-GNSS. On the other hand, performance evaluation and analysis on the signal qualities of GNSS measurements such as carrier phase, pseudorange, modulation and dilution of precision aims to evaluate errors that may be contributed by various phenomena.

The phenomenas that contribute to carrier phase and pseudorange errors are multipath propagation, atmospheric propagation errors (ionosphere and tropospheric errors), satellite and receiver clock biases and receiver noise [32]. The effects of these phenomena were usually studied and analyzed by subjecting the GNSS receiver to operate in open, rural, suburban and urban scenarios. The tests can be conducted in both static and/or dynamic modes. In an open sky scenario, the analysis aims to ascertain the errors contributed by the geometric distribution of satellites and atmospheric propagation errors. On the other hand, suburban and urban scenarios are used mostly to study the multipath propagation errors.

Figure 3 illustrates the outcome of these two categories of performance evaluation. The outcome of the evaluation on signal characteristics and qualities leads to identification and quantifying of the error sources in both conventional and RTKGNSS. Subsequently, it guides the designing and development of algorithms, models and filters for signal processing at the device level. On the other hand, performance evaluation of GNSS output measurements provides position error residuals that can be modeled mathematically for post-processing and improvement of direct measurements from these GNSS devices.

In the aspect of signal processing, some researchers implemented development of models, algorithms and filters that processes the pseudo-range and carrier phase measurements of the GNSS signals that may be affected by various sources of errors. Guo et al. [17] developed a time differenced positioning filter to handle the receiver pseudorange noise in Xiaomi. The filter estimates the inter-epoch position variation (IEPV) with time differenced uncombined L1/E1 and L5/E5 carrier phase observations. Next, the state equation was constructed with IEPV to estimate the actual position of the receiver more accurately. The state equations are 
formed with uncombined L1/E1 and L5/E5 pseudo-range observations and tested under open sky and GNSS-degraded environment. In Zhang et al. [33], time correlations of unmodelled errors are estimated from the residuals of code and phase observations of the double differenced method from 11 different baselines. Next, exponent and quadratic models were proposed to fit the time correlations. Finally, the sequential adjustment method is used to compute baseline solutions taking into consideration time correlations. In a recent work, Hoffman et al. [32] mitigate undifferenced carrier phase errors using Gauss-Markov (GM) process. The authors also proposed a satellite position computation algorithm to improve errors due to the geometry distribution of satellites.

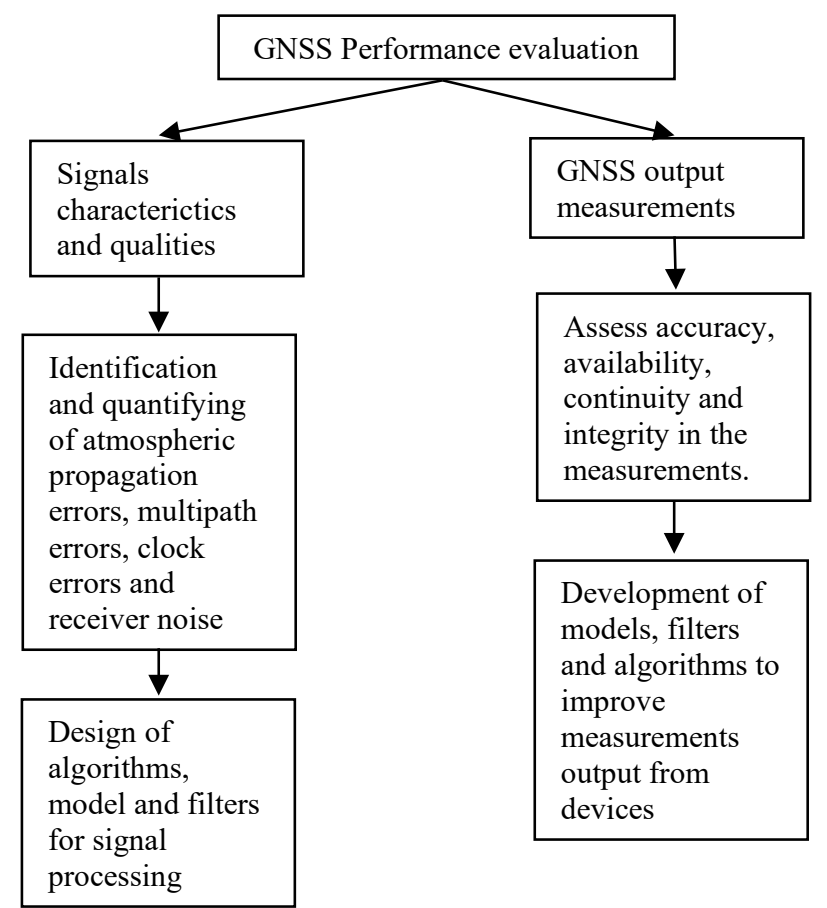

Fig. 3. Performance evaluation framework of GNSS receivers

GNSS position measurements could reveal error residuals when compared to ground truth data. These leads to the design of filter models to denoise random noise from measured outputs. For example, stationary GPS station position errors on seismic movements was modelled using an autoregressive moving average (ARMA) to de-noise the GPS time series data [34]. In another work, Tao and Bonnifait [35] modelled correlated biases in the L1-GPS using autoregressive model. The conventional L1-GPS errors was calculated based on comparison with ground truth and autocorrelation function reveals repeatable behaviour which could be modelled with AR(1) auto regressive filter.

In addition, our brief review on some applications of GP in section 3 of this paper has shown the recent applications of GP to process the positioning error residuals [30,31] from GNSS receivers. These GP models aim to model the measurement errors that may comprise of unmodelled corrrelated errors and random errors. In some works, GP was combined with $\mathrm{KF}$ to provide better estimates [23, 25].
Overall, the framework in Fig. 3 can be used for both conventional GNSS and RTK-GNSS. However, it should be noted that in the case of RTK-GNSS, atmospheric propagation errors, and clock biases may be cancelled out due to the relative positioning corrections from the RTK base station [32]. Hence, this reduce the error mitigation to handle multipath errors and random noise only.

\section{B. Findings and Discussion on Gaussian Process modeling of GNSS measurement errors}

The GP model is useful to process positioning errors for GNSS. A typical approach is to use the GP model to perform 'coarse' approximation of sensor measurements [22, 25, 26] using different kernel functions. Other researchers endeavour to obtain a GP with optimized hyperparameters that fits the data and noise well [24, 30,31]. The latter can be illustrated by the conceptual framework shown in Fig. 4.

Figure 4 shows that initial evaluation of conventional and RTK-GNSS is conducted. This evaluation is needed to evaluate positional drifts and the general performance of the GNSS due to multipath and non-multipath effects. Subsequently, training and testing data can be collected over a period of time using a conventional or RTK-GNSS rover station operating on static mode. Data-preprocessing may be needed to remove outliers so that data that exceed some threshold are removed.

Figure 4 illustrates that model development can be described by (1) where $y$ is the output; $f(X)$ is the GP prior model and $\varepsilon$ is the noise model. In modelling the accompanying noise model; the correlated and uncorrelated noise can be described as Gaussian noise. Next, training data is used to train the initial GP prior. The hyperparameters of the GP prior with noise model needs to be optimized to obtain a well fitted model. In the testing phase, test datasets are used as inputs to the trained model. In the final step; outputs from the trained model are reevaluated to assess the model performance.

Some general mathematical derivations will be used in this section to explain the framework illustrated in Fig. 4. Based on (3) and (4), the posterior of the corresponding function $f$ with given inputs and outputs $\{X, y\}$ is obtained using Bayes rules as defined by (5).

$$
p(f \mid X, y)=\frac{p(y \mid f) p(f \mid X)}{p(y \mid X)}
$$

where $\mathrm{p}(y \mid f)$ is the observation likelihood, $\mathrm{p}(f \mid X)$ denotes the prior distribution likelihood and the denominator is the marginal likelihood, $p(y \mid X)=\int \mathrm{p}(y \mid f) \mathrm{p}(f \mid X) \mathrm{df}$. Hence, the distribution of a particular function $f_{*}=f\left(x_{*}\right)$ with novel input $x_{*}$ can be obtained from (5) by averaging the outputs of all latent function values w.r.t. the posterior. This predictive distribution is shown in (6).

$$
\mathrm{p}\left(f_{*} \mid X, x_{*}, y\right)=\int \mathrm{p}\left(f_{*} \mid f, X, x_{*}\right) \mathrm{p}(f \mid X, y) \mathrm{df}
$$

Finally, the predicted distribution of the predicted output $y_{*}$ is obtained by marginalizing over $f_{*}$ as shown in (7).

$$
\mathrm{p}\left(y_{*} \mid X, x_{*}, y\right)=\int \mathrm{p}\left(y_{*} \mid f_{*}\right) \mathrm{p}\left(f_{*} \mid X, x_{*}, y\right) \mathrm{df}
$$




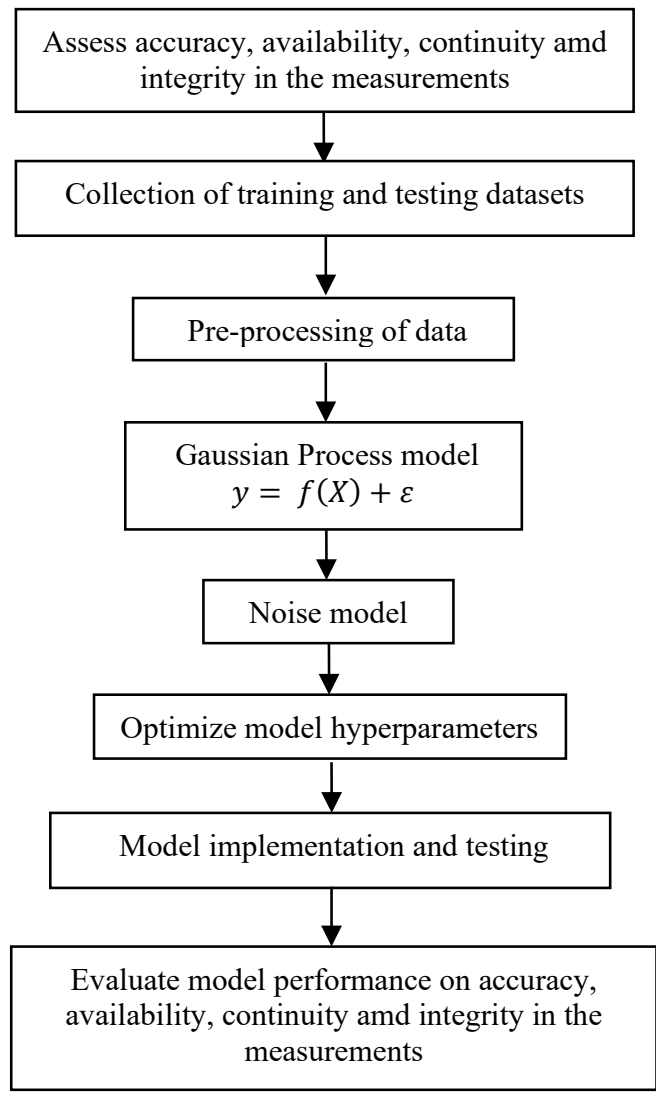

Fig. 4. Conceptual framework of GP modeling of errors in GNSS measurements

Equations (6) and (7) can be computed in closed-form as the observation likelihood and the posterior are both Gaussian [36]. Subsequently, the hyperparameters $\alpha$ of the kernel function $K$ and the observation noise $\sigma_{\mathrm{n}}^{2}$ could be estimated by maximizing the marginal log-likelihood shown in (8).

$$
\left\{\hat{\alpha}, \hat{\sigma}_{n}^{2}\right\}=\underset{\alpha, \sigma_{n}^{2}}{\operatorname{argmax}} \log p(y \mid X)
$$

The noise $\varepsilon$ could be described as i.i.d Gaussian distribution with zero mean and variance $\sigma_{n}$ in (9).

$$
\varepsilon \sim \mathcal{N}\left(0, \sigma_{n}\right)
$$

Equation (9) assumed white noise with variance $\sigma_{n}$. However, the GNSS is not only disturbed by white noise. Extensive research on noise affecting the GNSS measurements can be categorized to flicker noise which can be described by Brownian motion [37-40], band-pass process [41] and first order GaussMarkov process [31, 41, 42]. For example, our review on Hines and Hetland [31] adopted FOGM as the noise model. The FOGM is included in estimating the GP prior. As the FOGM is Gaussian, the predicted distribution could be computed in closed-form and is tractable. The hyperparameters of the covariance function were subsequently estimated using maximum likelihood method to reveal an optimized model. In [30], the authors also assumed the noise is Gaussian and utilized a reweighted least square method to optimize the GP model hyperparameters.
It is demonstrated here that by fitting a Gaussian noise model, will render the (6) and (7) to be computed in closed-form. To the best of our knowledge, the GNSS noise has only been approached using Gaussian models [30, 31]. He at al. [40] show that flicker noise consititute almost $90 \%$ of the noise in conventional GPS receivers. However, modeling flicker noise requires a reference time at which the process begin and may be infeasible to be incorporated in GP [31]. In another work, Langbein [38] investigated noise in GPS displacement measurement in two GPS networks in the United States and discovered that the noise in GPS measurement is a combination of various noise sources such as flicker noise, random-walk noise, power law noise and first order Gauss-Markov noise. Due to the complexity of identifying the best noise model; fitting the best noise model in a GP is a tedious process and computationally expensive.

\section{Research gaps and Recommendations for Future Research based on Gaussian Process}

The previous sections presented the conceptual framework on dealing with GNSS measurements based on Gaussian process. Most works assumed Gaussian noise models and attempted to solve a closed form of the GP to reach an optimized model that could fit the data well. To our knowledge, the noise model has not been studied by assuming a nonGaussian distribution. Secondly, applications of other GP methods in modelling GNSS errors have not been explored and extensively evaluated. Hence, this leads us to the following research questions:

a) Does the GNSS residuals fit a non-Gaussian distribution?

b) If the answer to question (a) is yes; is the GNSS measurements best modelled using generalized Gaussian process models (GGPM)?

c) Can Deep Gaussian process (DGP) provides a better solution that fits the data well?

\section{i) Generalized Gaussian Process Models (GGPM)}

The research questions posed in (a) and (b) brings us to revisit one area of GP namely generalized Gaussian process models (GGPM). The GGPM can be described as a Bayesian approach to Generalized Linear Models (GLM) [43]. The GGPM follows the formulation of GLM which can be specified as follows [44]:

$$
\begin{gathered}
\eta(x) \sim \mathcal{G P}\left(0, k\left(x, x^{\prime}\right)\right), \\
y \sim p(y \mid \theta, \phi), \text { and } g(\mathrm{E}[T(y) \mid \theta])=\eta(x)
\end{gathered}
$$

where $\eta(x)$ is the latent function modelled with a GP prior using $x$ as the function inputs and $y$ as observed outputs; $p(y \mid \theta, \phi)$ is a component that models the output with exponential family distribution with parameter $\theta$ and dispersion $\phi$ and $g(\mathrm{E}[T(y) \mid \theta])=\eta(x)$ is the link function that relates the mean of the sufficient statistics $T(y)$ with the latent function. This allow direct specifications of the relationship between the output mean and the latent function $\eta(x)$. This directly relates the latent function with various non-Gaussian models such as 
Gamma, inverse Gaussian, Poisson, Laplace, Binomial, etc. Hence, the observation likelihood $p(y \mid \theta, \phi)$ is no longer Gaussian. As a result, the posterior and predictive distribution (as denoted in (6) and (7)) is no longer tractable and could not be computed in closed form. To solve this, several approximate inference algorithms, such as Markov-chain Monte Carlo (MCMC) [45], variational bounds [46], Laplace approximation [47], and expectation propagation (EP) [48, 36] have been developed.

Recent researches had specified noise in GNSS receivers as non-Gaussian [49, 50]. Hence, revisitation of GGPM with approximate inference algorithms may be a promising modelling solution for GNSS measurement errors with nonGaussian distribution.

\section{ii) Deep Gaussian Process (DGP)}

The DGP was introduced by Damianou and Lawrence [51]. DGP is the distribution over functions constructed by multi layer of GPs. Each function is assumed to be drawn independently from GP priors [52]:

$$
\begin{gathered}
\mathrm{f}^{(1: \mathrm{L})}(\mathrm{x})=\mathrm{f}^{(\mathrm{L})}\left(\mathrm{f}^{\mathrm{L}-1)}\left(\ldots . . \mathrm{f}^{(2)}\left(\mathrm{f}^{(1)}(\mathrm{x})\right) \ldots . .\right)\right. \\
\text { where } f_{d}^{(l)} \sim \mathcal{G} \mathcal{P}\left(0, k_{\mathrm{d}}^{(l)}\left(x, x^{\prime}\right)\right) \text { for } f_{d}^{(l)} \in f^{(l)}
\end{gathered}
$$

Equation (11) is a DGP with one-dimensional input $x$. The number of layers is denoted by $L$. In each layer, each GP is allowed to have different hyperparameters. Hence, different covariance matrix corresponds to different kernels. Due to exponential scaling of the terms, the DGP is analytically intractable and may need approximation inference methods [53].

The DGP can overcome the limitations of the single-layer GP while retaining its advantages. In addition, DGP provides good uncertainty estimates, powerful non-linear mapping ability and great generalization capability [54]. The authors in [54] further enhanced the DGP for multi-view representation learning. Recently, Ye at al. [55] applied DGP for performance improvement of position and orientation data during GPS oustages. However, to the best of our knowledge, the DGP has not been employed for the modelling of GNSS measurements. Hence, the DGP can possibly address research question (c). This may be an area that can be explored in future works.

\section{CONCLUSION}

To this end, this paper has reviewed related works on performance evaluation and analysis of both conventional and RTK-GNSS. Recent and previous works on Gaussian process models on estimating and improving positioning errors were summarized and critically appraised. This leads to a unifying conceptual overview to show the readers various methods to approach performance evaluation and GP models established thus far by others. Consequently, a few research gaps were identified. Based on these research gaps, the authors propose future works to explore modeling and improvement errors in GNSS measurements using GGPM (that assumes non-Gaussian errors) and the DGP that could provide good uncertainty estimates and powerful non-linear mapping ability.

\section{ACKNOWLEDGMENT}

The authors wish to exress their appreciation to the Ministry of Education Malaysia for funding this research through the Fundamental Research Grant Scheme (FRGS) no: FRGS/1/ 2019/TK04/UITM/02/38.

\section{REFERENCES}

[1] F. De Ponte Müller (2017). Survey on ranging sensors and cooperative techniques for relative positioning of vehicles. Sensors (Switzerland), 17(2), pp.1-27.

[2] L. Du, "Cooperative Vehicle Localization Base on Extended Kalman Filter In Intelligent Transportation System," in 2019 28th Wirel. Opt. Commun. Conf., 2019, pp. 1-5

[3] M.A. Hossain, I. Elshafiey and A. Al-Sanie, "Cooperative vehicular positioning with VANET in urban environments," in 2016 IEEE AsiaPacific Conf. Appl. Electromagn. APACE 2016, 2016, pp.393-396

[4] Compare GNSS, Surveying News, Nov 5, 2016. Accessed on: April 10, 2021. [Online]. Available: https://surveyingnews.wordpress.com/tag/compare-gnss/

[5] B. Eissfeller, G. Ameres, V. Kropp and D. Sanroma, "Performance of GPS, GLONASS and Galileo," in Photogrammetric Week 2017, Wichmann Verlag, Heidelberg, 2017, pp. 185-199.

[6] HSU Geospatial Curriculum. Geospatial curriculum: Mobile Mapping \& GIS Terminology. [Online]. Available: http://gis.humboldt.edu/club/old_website/Images/Documents/MobileMa pping_Terminology.pdf

[7] "NovAtel's OEMV-1DFTM L1/L2 Receiver Provides RTK Positioning for Space-Constrained Applications," white paper, Novatel Inc., 2010. [Online]. Available: https://www.novatel.com/support/knowledgeandlearning/published-papers-and-documents/white-papers/. Retrieved 2 February 2019.

[8] D.Gebre-Egziabher, "Evaluation of Low-Cost, Centimeter-Level Accuracy OEM GNSS Receivers," Department of Aerospace Engineering and Mechanics, University of Minnesota, Research Project Final Report 2018-10, 2018.

[9] T. Yomchinda, "A method of multirate sensor fusion for target tracking and localization using extended Kalman Filter," in Proc. 4th Asian Conf. Def. Technol. ACDT 2017, vol. 2018-January, 2017, pp.1-7

[10] Z. Li, D. Zhou and Y. Huang, "Design of Outdoor Following Vehicle System Based on GPS-INS Fusion Navigation Algorithm," in Proc. 2018 2nd IEEE Adv. Inf. Manag. Commun. Electron. Autom. Control Conf. IMCEC 2018, 2018, pp.1285-1289.

[11] X. Zhu, W. Tian, G. Li and J. Yu, "Research on Localization Vehicle Based on Multiple Sensors Fusion System," in Proc. - 2017 Int. Conf. Comput. Network, Electron. Autom. ICCNEA 2017, 2017, pp.491-494.

[12] European Global Navigation Satellite System, What is GNSS?, Nov 19, 2020. Accessed on: April 10, 2021. [Online]. Available: https://www.gsa.europa.eu/european-gnss/what-gnss

[13] X. Luo, B. Richter and A. Cole, "Glonas only and Beidou only positioning," Technical Literature, Leica Geosystems AG Switzerland Heerbrugg, Schweiz, 2014.

[14] T. Baybura, İ. Tiryakioğlu, M. A. Uğur, H. İ. Solak, Ş. Şafak (2019). Examining the Accuracy of Network RTK and Long Base RTK Methods with Repetitive Measurements. Journal of Sensors, vol. 2019. https://doi.org/10.1155/2019/3572605

[15] H. Chengyan, G. Ji, L. Xiaochun and L. Jun, "Multipath performance analysis of GNSS navigation signals," in 2014 IEEE Workshop on Electronics, Computer and Applications, 2014, pp. 379-382, doi: 10.1109/IWECA.2014.6845636.

[16] M. Rychlicki, Z. Kasprzyk, A. Rosiński (2020). Analysis of Accuracy and Reliability of Different Types of GPS Receivers. Sensors 2020, 6498. https://doi.org/10.3390/s20226498

[17] L. Guo, F. Wang, J. Sang, X. Lin and X. Gong and W. Zhang. (2020). Characteristics Analysis of Raw Multi-GNSS Measurement from Xiaomi Mi 8 and Positioning Performance Improvement with L5/E5 Frequency in an Urban Environment. Remote Sens. 2020, 12(4). https://doi.org/10.3390/rs12040744

[18] P. Crosta, P. Zoccarato, R. Lucas and G. Pasquale, "Dual Frequency Mass-market Chips: Test Results and Ways to Optimize PVT Performance," in Proceedings of the 31st International Technical Meeting of The Satellite Division of the Institute of Navigation (ION GNSS+ 2018), Miami, FL, USA, 24-28 September, 2018. 
[19] S.K. Moore (2017). Super-accurate GPS coming to smartphones in 2018. IEEE Spectr. 2017, 54, pp. 10-11.

[20] Z. He, Y. Hu, J. Wu, J. Wang, J. Hou and K. Wang, "A comprehensive method for multipath performance analysis of GNSS navigation signals," in 2011 IEEE International Conference on Signal Processing, Communications and Computing (ICSPCC), 2011, pp. 1-6, doi: 10.1109/ICSPCC.2011.6061564.

[21] L. Cheng, S. Ramchandran and T. Vatanen. (2019). An additive Gaussian process regression model for interpretable non-parametric analysis of longitudinal data. Nat Commun 10, 1798 . https://doi.org/10.1038/s41467-019-09785-8

[22] J. Hidalgo-Carrió, D. Hennes, J. Schwendner and F. Kirchner, "Gaussian process estimation of odometry errors for localization and mapping," in 2017 IEEE International Conference on Robotics and Automation (ICRA), Singapore, 2017, pp. 5696-5701.

[23] V. Kurtz and H. Lin, "Kalman Filtering with Gaussian Processes Measurement Noise", arXiv:1909.10582 [stat.ML], Cornell University, 2019

[24] C. E. Rasmussen, "Gaussian processes in machine learning", in Advanced lectures on machine learning, Springer, 2004, pp. 63-71.

[25] W. Ye, Z. Liu, C. Li, and J. Fang (2018). Enhanced Kalman Filter using Noisy Input Gaussian Process Regression for Bridging GPS Outages in a POS. The Journal of Navigation, 71, pp. 565-584.

[26] L. Kortesalmi, "Gaussian Process Regression based GPS Variance Estimation and Trajectory Forecasting," Master thesis, Department of Computer and Information Science, Linköping University, 2018.

[27] M. Tiger and F. Heintz, "Towards Unsupervised Learning, Classification and Prediction of Activities in a Stream-Based Framework," in Proceedings of the Thirteenth Scandinavian Conference on Artificial Intelligence (SCAI), 2015. ISBN: 978-1-61499-588-3.

[28] M. Tiger and F. Heintz, "Online sparse Gaussian process regression for trajectory modeling," in 2015 18th International Conference on Information Fusion (Fusion). July 2015, pp. 782-791.

[29] M. Tiger and F. Heintz, "Gaussian Process Based Motion Pattern Recognition with Sequential Local Models," in 2018 IEEE Intelligent Vehicles Symposium (IV), 2018.

[30] M. Lin, X. Song, Q. Qian, H. Li, L. Sun, S. Zhu, R. Jin, "Robust Gaussian Process Regression for Real-Time High Precision GPS Signal Enhancement", Applied Data Science Track Paper KDD 2019, Anchorage, AK, USA, August, 2019, pp. 2838-2847.

[31] T. T. Hines and E. A. Hetland. (2018). Revealing transient strain in geodetic data with Gaussian process regression. Geophysical Journal International, 212(3), pp. 2116-2130.

[32] M. Hoffmann, P. Kryszkiewicz and G.P. Koudouridis (2020). Modeling of Real Time Kinematics localization error for use in $5 \mathrm{G}$ networks. Journal of Wireless Com Network 2020, 31. https://doi.org/10.1186/s13638-020-1641-8

[33] Z. Zhang, B. Li, Y. Shen (2017). Comparison and analysis of nmodelled errors in GPS and BeiDou signals. Geodesy and Geodynamics, 8(1), pp. 41-48. https://doi.org/10.1016/j.geog.2016.09.005.

[34] J. Li, K. Miyashita, T. Kato and S. Miyazaki (2000). GPS time series modeling by autoregressive moving average method: Application to the crustal deformation in central Japan. Earth Planets Space, 52, pp. 155162.

[35] Z. Tao and P. Bonnifait, "Modeling L1-GPS Errors for an Enhanced Data Fusion with Lane Marking Maps for Road Automated Vehicles," European Navigation Conference (ENC 2015), France, 2015.

[36] C. E. Rasmussen and C. K. I.Williams, "Gaussian Processes for Machine Learning," MIT Press, 2006.

[37] S.D.P. Williams, Y. Bock, P. Fang, P. Jamason, R.M. Nikolaidis, L. Prawirodirdjo, M. Miller and D.J. Johnson (2004). Error analysis of continuous GPS position time series. J. geophys. Res., 109(B3), pp. 119.

[38] J. Langbein, (2008). Noise in GPS displacement measurements from Southern California and Southern Nevada. J. geophys. Res., 113(5), pp. $1-12$.

[39] J.R. Murray and.J. Svarc (2017). Global Positioning System data collection, processing, and analysis conducted by the U.S. Geological Survey Earthquake Hazards Program. Seismol. Res. Lett., 88(3), pp. 1-10.

[40] X. He, J.P. Montillet, R. Fernandes, M. Bos, K. Yu, X. Hua and W. Jiang (2017). Review of current GPS methodologies for producing accurate time series and their error sources. J. Geodyn., 106, pp. 12-29.

[41] J. Langbein (2004). Noise in two-color electronic distance meter measurements revisited. J. geophys. Res., 109(4), pp. 1-16.
[42] K. Dmitrieva, P. Segall and C. DeMets (2015). Network-based estimationof time-dependent noise in GPS position time series. Journal of Geodesy, 89(6), pp. 591-606.

[43] P. McCullagh and J.A. Nelder, "Generalized linear models.," Chapman \& Hall, CRC, 1989. ISBN 9780412317606.

[44] L. Shang and A.B. Chan, "On Approximate Inference for Generalized Gaussian Process Models", arXiv e-prints, 2013.

[45] H. Nickisch and C. E. Rasmussen (2008). Approximations for binary Gaussian process classification.. Journal of Machine Learning Research, 9, pp. 2035-2078.

[46] M. Gibbs and D. J. C. Mackay (2000). Variational Gaussian process classifiers. IEEE Transactions on Neural Networks, 11, pp. 1458-1464.

[47] C. K. I. Williams and D. Barber (1998). Bayesian classification with Gaussian processes. IEEE Transactions on Pattern Analysis and Machine Intelligence, 20(12), pp.1342-1351.

[48] T. P. Minka, "A family of algorithms for approximate Bayesian inference.," PhD thesis, Massachusetts Institute of Technology, 2001.

[49] V. Havyarimana, Z. Xiao, P. C. Bizimana, D. Hanyurwimfura and H. Jiang (2021). Toward Accurate Intervehicle Positioning Based on GNSS Pseudorange Measurements Under Non-Gaussian Generalized Errors," IEEE Transactions on Instrumentation and Measurement, 70, pp. 1-12. Art no. 1001112, doi: 10.1109/TIM.2020.3016707.

[50] L. Zhao, J. Zhang, L. Li, F. Yang, X. Liu (2020). Position-Domain NonGaussian Error Overbounding for ARAIM. Remote Sensing. 2020, 12(12). https://doi.org/10.3390/rs12121992

[51] A. C. Damianou and N. D. Lawrence, "Deep Gaussian processes," in 16th International Workshop on Artificial Intelligence and Statistics, 2013.

[52] D. Duvenaud., "Automatic model construction with Gaussian processes," $\mathrm{PhD}$ thesis, University of Cambridge, 2014.

[53] K. Vafa, "Training deep Gaussian processes with sampling," in 30th Conference on Neural Information Processing Systems (NIPS 2016), Barcelona, Spain, 2016.

[54] S. Sun, W. Dong and Q. Liu, "Multi-view Representation Learning with Deep Gaussian Processes," in IEEE Transactions on Pattern Analysis and Machine Intelligence, doi: 10.1109/TPAMI.2020.3001433.

[55] W. Ye, B. Wang, Y. Liu, B. Gu and H. Chen (2020). Deep Gaussian Process Regression for Performance Improvement of POS During GPS Outages. IEEE Access, 8, pp. 117483-117492. doi: 10.1109/ACCESS.2020.3004706.

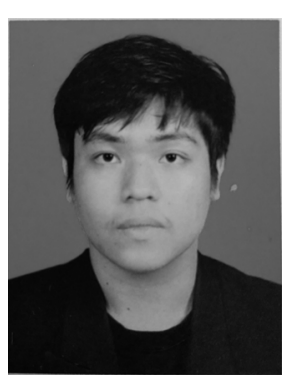

Ravenny Sandin Nahar graduated with Bachelor of Engineering (Hons.) Electronic Enginnering at the Faculty of Electrical Engineering, Universiti Teknologi Mara (UiTM) in 2019. He is currently pursuing his post graduate studies in Master in Engineering (Electrical Engineering) at the School of Engineering, College of Engineering, Universiti Teknologi MARA, Shah Alam. His research interest includes artificial intelligence and smart systems.

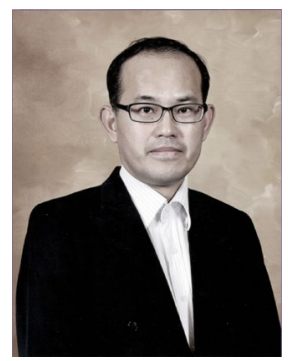

Kok Mun Ng received the B.Eng and M.Eng degrees in systems engineering from the Universiti Tun Hussein Onn, Malaysia, in 2002 and 2006 respectively. He obtained the Ph.D. degree in Systems Engineering from Universiti Kebangsaan Malaysia, Bangi, Malaysia in 2016. From 2002 to 2003 , he worked as a system engineer at Matromatics Handling Systems, Malaysia. He was with Stamford College, Malaysia from 2006 to 2008 as a lecturer before joining the Faculty of Electrical Engineering, Universiti Teknologi MARA, where he works as a senior lecturer at the Systems Engineering department. He is the author of more than 30 articles and conference papers. His research interests include system 
modeling and optimization, articial intelligence, intelligent transportation systems, mapping and localization. He is a subeditor of the Journal of Electrical \& Electronic Systems Research (JEESR) and a research fellow at the university vehicle telematics industrial research lab.

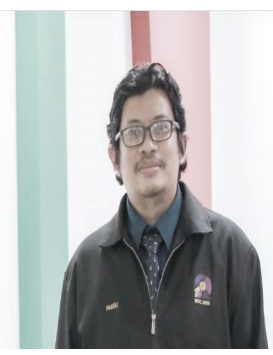

Noorfadzli Abdul Razak completed his $\mathrm{PhD}$ in Electrical Engineering at the Faculty of Electrical Engineering, Universiti Teknologi MARA (UiTM) in 2019. He received his Masters in Electrical Engineering (Mechatronic and Automatic Control) at the Faculty of Electrical Engineering, Universiti Teknologi Malaysia (UTM) in 2017. In 2004, he obtained a Bachelor of Electrical Engineering from Kolej Universiti Teknologi Tun Hussein Onn (KUiTTHO). He started his carrier as a Vocational Training Officer in Kolej Kemahiran Tinggi MARA, Balik Pulau from 2005 to 2008. Then, he works as a lecturer in the Faculty of Electrical Engineering, Universiti Teknologi Melaka Malaysia (UTeM). In 2009, he joined the Faculty of Electrical Engineering, UiTM. At present, he is a senior lecturer in the faculty and has been appointed to lead an Innovative Electro Mobility (ITEM) research lab. He actively conducts research and development in autonomous control, mobile robot, automation system and electric vehicle. He had published more than 30 articles and conference papers in the respective field.

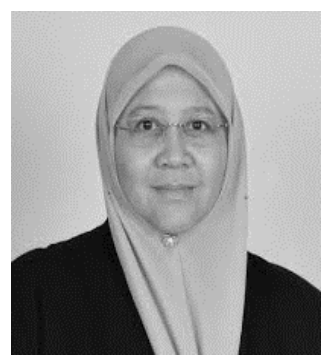

Juliana Johari received $\mathrm{PhD}$ in Micro-Engineering and NanoElectronics from Universiti Kebangsaan Malaysia, Master degree in Biomedical Engineering from University of Surrey, United Kingdom and Bachelor in Electrical and Electronics Engineering from University of Strathclyde, United Kingdom. She is currently an Associate Professor in Control System and Instrumentation Engineering at the School of Electrical Engineering, Universiti Teknologi MARA Malaysia and Affliate Professor in Electronics and Instrumentation Engineering at the B.S. Abdur Rahman Crescent Institute of Science and Technology, Chennai, India. Area of specialization including robotics, automation, MEMS and Artificial Intelligence. 